\title{
The Development and Design of the Exploration on the Interaction between Adrenaline with Fatty Acid: A Comprehensive Student Experimental Project
}

\author{
Benzhen Xu, Dongcheng Ren and Tao Liu* \\ Department of Chemistry and Chemical Engineering, Jining University, Qufu 273155, Shandong, \\ China \\ *liutao_2005@126.com
}

Keywords: Cyclic voltametric; Adrenaline; Fatty acid

\begin{abstract}
Putting computers and large precise instruments into the teaching of structural chemistry, designing and developing comprehensive experiment, and revealing learning level of structure-property-application, will contribute to enhance students the learning interesting on theoretical knowledge, exercise students the ability to find problem and solve problem, and cultivate students the scientific thought and scientific literacy.
\end{abstract}

\section{Introduction}

Adrenaline belongs to a group of compounds known as catecholamines that plays a particularly important role in the regulation of physiological process in living systems [1]. It can be oxidized easily and the product of electro oxidation is adrenaline Quinone [2]. Adrenaline cannot be dissolved in water and organic solvents itself, but the protonated adrenaline will be dissolved in the solvent that can donor proton. Adrenaline plays a central role in the short-term stress reaction, the physiological response to conditions that threaten the physical integrity of the body. Adrenaline can be studied directly by electrochemical methods because of its structural similarity to o-dihydroxybenzene, and the $-\mathrm{CH}(\mathrm{OH})-$ group at the $\alpha$ carbon facilitates the easy donation of an electron [3-10].

\section{Experimental}

The reagent of adrenaline (>97\%) was supplied by Fluka Co. (Sweden). The concentration of adrenaline aqueous solution was $6 \times 10^{-3} \mathrm{~mol} / \mathrm{L}$. $\mathrm{KCl}-\mathrm{HCl}$ solution was used as the studying medium with constant ionic strength $(\mathrm{I}=1)$ of $\mathrm{KCl}$. Other employed solutions were prepared with analytic grade reagents and doubly distilled water.

Cyclic voltammetry was performed on an EG\&G PAR M398 electrochemical impedance system with an M283 potentiostat/galvanostat. The three-electrode-system was used to carry out electrochemical tests. A platinum circular electrode and a graphite electrode served as a working electrode, respectively, a platinum wire served as a counter electrode, and a saturation calomel electrode (SCE) served as reference electrode. A Luggin capillary was used to connect the reference and working electrodes. Highly pure nitrogen gas was passed through the solution for $10 \mathrm{~min}$ to remove oxygen dissolved in solution before measurements, and all measurements were carried out under nitrogen atmosphere at room temperature $\left(25.0 \pm 0.1^{\circ} \mathrm{C}\right)$.

\section{Results and discussion}

The $\mathrm{CV}$ curves of $6 \times 10^{-3} \mathrm{~mol} / \mathrm{L}$ adrenaline at platinum electrode in the $\mathrm{KCl}-\mathrm{HCl}$ solution (constant ionic strength ( $\mathrm{I}=1)$ and constant $\mathrm{PH}$ value is 1$)$ with different concentration of formic acid are presented in Fig. 1. Peak 1 of curve a corresponds to the oxidation of adrenaline into adrenalinequinone (anodic peak), and Peak 2 of curve a corresponds to the reduction of adrenalinequinone into adrenaline (canodic peak). It can be seen that with the addition of formic acid, the electron transfer ability of adrenaline 
decreases as follows: the anodic peak potential $\left(E_{\mathrm{pa}}\right)$ shifts positively, the canodic peak potential $\left(E_{\mathrm{pc}}\right)$ shifts negatively, the peak-to-peak potential separation between anodic and canodic peak potential $\left(\Delta E_{\mathrm{p}}\right)$ increases, and the anodic and canodic peak current $\left(i_{\mathrm{pa}}\right.$ and $\left.i_{\mathrm{pc}}\right)$ decrease significantly. The results demonstrate the inhibition effect of formic acid on the electron transfer reaction of adrenaline, which has been verified by the fact that formic acid can form stable supramolecular complexes with adrenaline by hydrogen bond interaction and the formed supramolecular complexes will protect the phenolic hydroxyl groups of adrenaline and make it hard to donate $\mathrm{H}^{+}$and be oxidized [12].

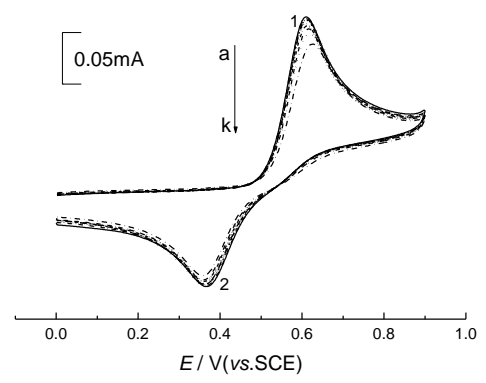

Figure 1. $\mathrm{CV}$ curves of $6 \times 10^{-3} \mathrm{~mol} / \mathrm{L}$ adrenaline at platinum electrode in $\mathrm{KCl}-\mathrm{HCl}(\mathrm{pH}=1)$ solution with different concentration of formic acid. Scan rate: $100 \mathrm{mV} / \mathrm{s}$.

$$
C_{\text {adrenaline }}: C_{\text {formic acid }} \text { (a) 1:0; (b) 1:1; (c) } 1: 2 ; \text { (d) 1:3; (e) 1:10; (f) 1:20; (g) 1:50; (h) 1:100; (i) 1:200; (j) }
$$
1:500; (k) 1:750

The CV curves of $6 \times 10^{-3} \mathrm{~mol} / \mathrm{L}$ adrenaline at platinum electrode in a series of $\mathrm{KCl}-\mathrm{HCl}$ solution (constant ionic strength $(\mathrm{I}=1)$ of $\mathrm{KCl}$ ) with different high concentrations of formic acid and different $\mathrm{PH}$ values are shown in Fig. 2. It can be seen from Fig. 2 that with the concentration of formic acid increasing and the $\mathrm{PH}$ value of solution decreasing, the peak-to-peak potential separation between anodic and canodic peak potential becomes larger, while the anodic and canodic peak current becomes smaller. In this experimental condition, there is a linear relationship between $E_{\mathrm{pa}}$ (and $\left.i_{\mathrm{pa}}\right)$ with PH value.

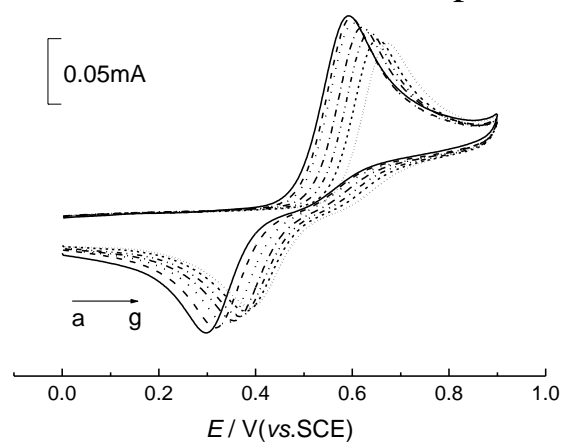

Figure 2. $\mathrm{CV}$ curves of $6 \times 10^{-3} \mathrm{~mol} / \mathrm{L}$ adrenaline at platinum electrode in a series of $\mathrm{KCl}-\mathrm{HCl}$ solution with different high concentrations of formic acid and different PH values. Scan rate: $100 \mathrm{mV} / \mathrm{s}$.

pH: (a) 1.84; (b) 1.59; (c) 1.34; (d) 1.00; (e) 0.71 ; (f) 0.43 ; (g) 0.16 .

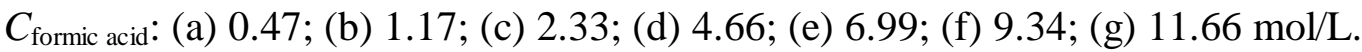

In order to eliminate the influence of $\mathrm{PH}$ values on the experimental results, we drew the $\mathrm{CV}$ curves of $6 \times 10^{-3} \mathrm{~mol} / \mathrm{L}$ adrenaline at graphite electrode in the $\mathrm{HCl}$ solution (constant ionic strength) with different $\mathrm{PH}$ values (see Fig. 3). We can find that in the $\mathrm{HCl}$ solution without formic acid, $\Delta E_{\mathrm{p}}$ decreases and $i_{\mathrm{p}}$ increases with the $\mathrm{PH}$ value becoming smaller, which is contrary to the changing trend of $\Delta E_{\mathrm{p}}$ and $i_{\mathrm{p}}$ for adrenaline in the solution with different high concentrations of formic acid and different $\mathrm{PH}$ values. The phenomenon can be interpreted by the hydrogen bond interaction between adrenaline and formic acid, which will protect the phenolic hydroxyl groups of adrenaline and make it hard to donate $\mathrm{H}^{+}$and be oxidized. The effect of hydrogen bond interaction on the adrenaline is much larger than the effect of $\mathrm{PH}$ value on it. Therefore, $\Delta E_{\mathrm{p}}$ will decrease and $i_{\mathrm{p}}$ will increase with the $\mathrm{PH}$ values increasing in the solution with different high concentrations of formic acid and different $\mathrm{PH}$ values. 


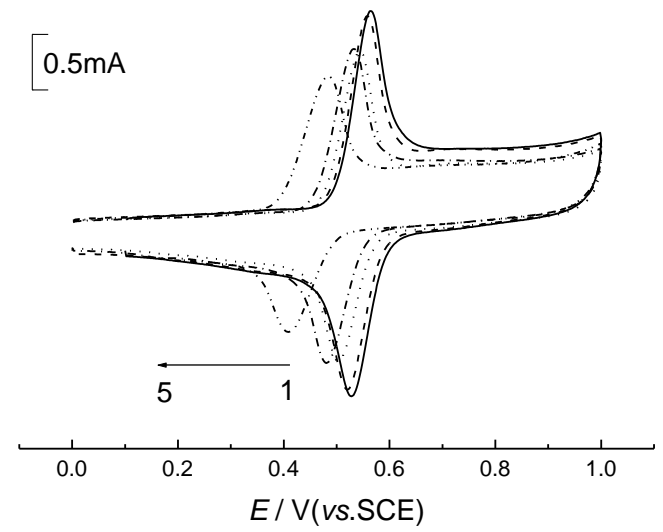

Figure 3. $\mathrm{CV}$ curves of $6 \times 10^{-3} \mathrm{~mol} / \mathrm{L}$ adrenaline at graphite electrode in the $\mathrm{HCl}$ solution with different $\mathrm{PH}$ values. Scan rate: $100 \mathrm{mV} / \mathrm{s}$.

$C_{\mathrm{HCl}}$ (1) 0.5 ; (2) 0.4 ; (3) 0.2 ; (4) 0.1 ; (5) $0.01 \mathrm{~mol} / \mathrm{L}$

\section{Summary}

We study the interaction of adrenaline with formic acid and acetic acid by CV approach. With the addition of formic acid and acetic acid, the electron transfer ability of adrenaline decreases, the peak-to-peak potential separation between anodic and canodic peak potential increases, and the anodic and canodic peak current decrease significantly. The $\mathrm{CV}$ curves of $6 \times 10^{-3} \mathrm{~mol} / \mathrm{L}$ adrenaline at platinum electrode in a series of $\mathrm{KCl}-\mathrm{HCl}$ solution with different high concentrations of formic acid (or acetic acid) and different $\mathrm{PH}$ values indicate that the effect of hydrogen bond interaction on the adrenaline is much larger than the effect of $\mathrm{PH}$ value on it.

\section{Acknowledgements}

This work was supported by the National Training Program of Innovation and Entrepreneurship for Undergraduates (Nos. 201610454011 and 201610454019)).

\section{References}

[1] M. D. Hawley, S. V. Tatawawadi, S. Piekarski, R. N. Adams. J. Am. Chem. Soc. 89 (1967) 447.

[2] H. Zheng, in Pharmaceutical Chemistry, People's Medical Publishing House, Beijing, 2003.

[3] A. Galal. J. Solid State Electrochem. 35 (1988) 277.

[4] S. H. Kim, J. W. Lee, I. H. Yeo. Electrochim. Acta 45 (2000) 2889.

[5] R. P. H. Nikolajsen, A. M. Hansena. Anal. Chim. Acta 449 (2001) 1.

[6] H. M. Zhang, X. L. Zhou, R. T. N. Hui, Q. Li, D. P. Liu. Talanta 56 (2002) 1081.

[7] Y. Z. Song, J. F. Zhou, Y. Song, Y. Wei, H. Wang. Bioorg. Med. Chem. Lett. 15 (2005) 4671.

[8] L. Wang, J. Bai, P. Huang, H. Wang, L. Zhang, Y. Zhao. Int. J. Electrochem. Sci. 1 (2006) 238.

[9] T. E. Graedel, C. J. Weschler. Rev. Geophys. Space Phys. 19 (1981) 505.

[10] R. V. Niquirilo, E. Teixeira-Neto, G. S. Buzzo, H. B. Suffredini. Int. J. Electrochem. Sci. 5 (2010) 344. 